\title{
The Effects of Emitter-Tied Field Plates on Lateral PNP
}

\section{SAND- $98-0625 \mathrm{C}$}

\section{Ionizing Radiation Response CONF-980705--}

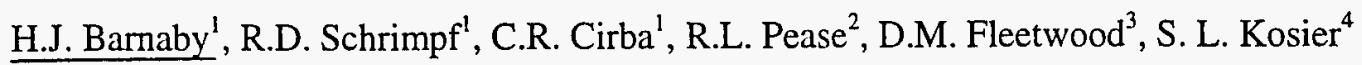

${ }^{1}$ Department of ECE, Vanderbilt University, Nashville, TN 37232

${ }^{2}$ RLP Research, Inc., Albuquerque, NM 87122

${ }^{3}$ Sandia National Laboratories, Albuquerque, NM 87185-1083

${ }^{4}$ VTC Inc. Bloomington, MN 55425-1350

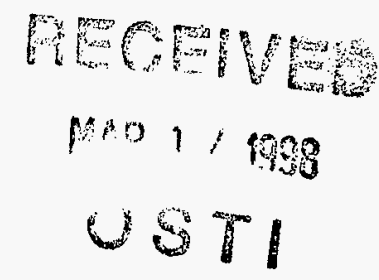

\section{ABSTRACT}

Radiation response comparisons of lateral PNP bipolar technologies reveal that device hardening may be achieved by extending the emitter contact over the active base. The emitter-tied field plate suppresses recombination of carriers with interface traps.

\section{ACKNOWLEDGMENTS}

This work is supported by DSWA. Work performed at Sandia National Laboratories was supported by DSWA and the U. S. Department of Energy through Contract No. DE-AC04-94A85000. The authors are grateful to Lew Cohn of DSWA and Ken Galloway of Vanderbilt University for their continuing support of this work. 


\section{DISCLAIMER}

This report was prepared as an account of work sponsored by an agency of the United States Government. Neither the United States Government nor any agency thereof, nor any of their employees, makes any warranty, express or implied, or assumes any legal liability or responsibility for the accuracy, completeness, or usefulness of any information, apparatus, product, or process disclosed, or represents that its use would not infringe privately owned rights. Reference herein to any specific commercial product, process, or service by trade name, trademark, manufacturer, or otherwise does not necessarily constitute or imply its endorsement, recommendation, or favoring by the United States Government or any agency thereof. The views and opinions of authors expressed herein do not necessarily state or reflect those of the United States Government or any agency thereof. 


\section{INTRODUCTION}

Lateral PNP (LPNP) bipolar junction transistors commonly exhibit a significant increase in base current upon exposure to ionizing radiation [1,2]. This response causes the current gain $\left(\beta=I_{C} / I_{B}\right)$ of the device to decrease. The primary mechanism responsible for radiation-induced excess base current is the buildup of traps near the $\mathrm{Si} / \mathrm{SiO}_{2}$ interface [1-5]. These traps provide recombination centers for free carriers traveling along the surface near the interface. Moreover, the silicon surface is the principal current path for LPNP devices operating in the forward active mode. Thus, the combined effects of increased traps and high free carrier densities at the $\mathrm{Si} / \mathrm{SiO}_{2}$ interface make the lateral structure extremely susceptible to radiation-induced gain degradation.

Data obtained from irradiated LPNP parts manufactured at VTC indicate that an emitter-tied field plate above the active base region may suppress recombination current at the $\mathrm{Si}_{\mathrm{SiO}}$ interface [2]. Here the active base is defined as the base region between the emitter-base and base-collector metallurgical junctions. A representational cross-section of a LPNP transistor is shown in Fig. 1. The crosshatched region illustrates the location of the emitter-tied field plate. After $100 \mathrm{krad}\left(\mathrm{SiO}_{2}\right)$ irradiation at a dose rate of $83 \mathrm{rad}\left(\mathrm{SiO}_{2}\right) / \mathrm{s}$, the VTC devices show less than $20 \%$ decrease in peak gain. This is significantly less degradation than observed in devices manufactured by Analog Devices (RF25) without an emitter-tied field plate [1].

The characteristics of the bipolar base oxide and base surface doping concentration are often identified as the most important physical parameters affecting ionizing radiation response [6]. The VTC and RF25 technologies have different doping concentrations, and capacitance-voltage measurements performed on VTC and RF25 MOS capacitors indicate that there is a significant difference in radiation-induced interface trap buildup [5]. These are two reasons for the relative hardness of the VTC devices. However, analysis of the experimental data suggests that the difference in gain degradation is not entirely due to these causes. Another reason appears to be the presence of the emitter-tied field plate in the VTC devices. Since information regarding the field plate can be obtained simply by analyzing the device layout, it is useful to examine the effectiveness of field plates in suppressing gain degradation.

\section{BACKGROUND}

The use of field plates to modify the surface potential in diodes and bipolar junction transistors has been the subject of numerous studies [2, 6-11]. Indeed, the model developed by Reddi illustrates how metal electrodes above the emitter-base junction in vertical npn devices can affect the current gain [7]. This theoretical model "was not developed for the ionizing radiation case but contains the necessary physical dependencies (surface potential and interface state density) for inclusion in ionizing radiation effects" [6]. Data obtained from gated diode and transistor structures were used in previous studies to identify and "separate" the mechanisms responsible for radiation-induced current gain in npn transistors $[3,6]$.

Most of the studies cited above concentrate specifically on the radiation response of npn transistors. The merit of using field plates to reduce radiation-induced gain degradation in LPNP transistors has not been reported for modern bipolar technologies. In this paper, a discussion of the physical mechanisms relating to the field plate and its effects on LPNP radiation response is presented in order to provide an analytical model of experimental results. The analysis is supported by computer simulations. In these simulations, the I-V characteristics of pre- and post-irradiation LPNP models are computed for field plates of varying lengths. The modeling suggests that emitter-tied metallization above the active base may suppress radiation-induced excess base current and can boost current gain. At present, the modeling does not account for the effect field plates may also have on the buildup of oxide charge or interface traps during irradiation. Previous studies indicate that field plates and other metallization runs can have an impact on the buildup of these charges and traps during irradiation [8-10]. The full paper will consider these effects in detail. The modeling results presented here provide another potential explanation for the relative radiation hardness of the VTC devices as compared to the devices examined previously [1], and suggest that devices with field plates may deserve extra attention for potential use in low dose rate environments.

\section{EXPERIMENTAL RESULTS}

The data presented in this paper are obtained from total dose experiments on two lateral bipolar technologies. Results taken from transistors manufactured as part of the RF25 development process from ADI were previously presented by Schmidt et al. [1]. The VTC devices considered in this paper are from a BiCMOS process, PolarMOS3 [2]. Both the RF25 and VTC devices were irradiated in a 10-keV x-ray source at Sandia 
National Laboratories. The parts were exposed at various dose rates (between 83 and $1670 \mathrm{rad}\left(\mathrm{SiO}_{2}\right) / \mathrm{s}$ ) with all terminals grounded to total doses up to $500 \mathrm{krad}\left(\mathrm{SiO}_{2}\right)$. The normalized current gain for one sample of each technology is plotted versus emitter-base voltage in Fig. 2. The figure shows gain characteristics for the parts prior to irradiation and after total dose exposure. After $100 \mathrm{krad}\left(\mathrm{SiO}_{2}\right)$, the peak current gain of the RF25 sample has degraded more than 70 percent, while the peak of the VTC sample has degraded less than 20 percent.

For both technologies, the radiation-induced changes in interface trap densities were measured from MOS capacitors. After $100 \mathrm{krad}\left(\mathrm{SiO}_{2}\right)$ exposure, the RF25 capacitors show an increase in trap density of more than $10^{11} \mathrm{~cm}^{-2}$, whereas the VTC devices show an increase of slightly more than $10^{10} \mathrm{~cm}^{-2}[2,5]$. This variation in interface trap buildup is a major reason for the difference in hardness between the two technologies. Another potential factor limiting gain degradation in the VTC transistors is the existence of a threshold adjust implant, which alters the doping concentration in the active base near the interface. Previous studies show that radiation response in transistors is a function of base surface doping [6]. However, these factors do not appear to be the only reasons for the differences in gain degradation. Analysis of LPNP structures with varying field plate lengths suggests that an extension of the emitter contact over the active base is also important.

\section{DEVICE MODELING}

In order to analyze the effects of emitter-tied field plates on LPNP radiation response, computer simulations were run on two-dimensional virtual test structures. Device modeling is performed with ATLAS from the SILVACO suite of simulation tools. Fig. 3 shows a cross-section of one of the test structures used in the analysis. This structure has an aluminum field plate extending over the active base region at a distance of $2.0 \mu \mathrm{m}$ from the emitter contact. The n-type base region is uniformly doped with a concentration of $10^{16} \mathrm{~cm}^{-3}$ and has an active width of $1.75 \mu \mathrm{m}$. A Gaussian distribution of acceptors, with a peak concentration of $10^{19} \mathrm{~cm}^{-3}$ at the interface, defines the emitter and collector regions.

Simulations of the device response prior to radiation exposure were performed with varying lengths of field plate. These lengths are: $0.0 \mu \mathrm{m}$ (no field plate), $1.0 \mu \mathrm{m}$, and $2.0 \mu \mathrm{m}$. Fig. 4 illustrates the effects of varying field plate length on current gain. The results indicate that as the length is increased, the pre-irradiation current gain is reduced, but only slightly.

In order to model the effects of emitter-tied field plates on the LPNP transistor's radiation response, interface parameters are added to the simulation to model the total dose mechanisms [1-4]. These parameters are: 1) surface recombination velocity (SRV) to model the increase in interface trap density and 2) a uniform layer of sheet charge at the interface $\left(\mathrm{N}_{\mathrm{Ox}}\right)$ to model the increase in trapped charge in the oxide. For these simulations SRV is set to $10^{4} \mathrm{~cm} / \mathrm{s}$ and $N_{O X}$ is set to $10^{11} \mathrm{~cm}^{-2}$. Previous studies on modeling radiation effects in bipolar devices confirm that these interface parameter values are reasonable approximations for total doses of approximately $100 \mathrm{krad}\left(\mathrm{SiO}_{2}\right)$ [1-4]. Fig. 5 illustrates the effects of the same three field plate lengths on current gain with the added charge densities. Unlike the pre-irradiation modeling, these results indicate that, as the length is increased, the current gain is increased. Indeed, a field plate extending almost entirely across the active base region can boost degraded current gain more than 60 percent above that obtained without a field plate.

\section{DISCUSSION}

Ionizing radiation-induced gain degradation in the LPNP transistor is due primarily to increased base current. This excess base current is caused by the recombination of carriers with radiation-induced interface traps. The majority of recombination takes place near the $\mathrm{Si} / \mathrm{SiO}_{2}$ interface within the emitter-base space charge region and the neutral base. The neutral base is defined as the region of active base between both the emitterbase and base-collector space charge regions.

According to Shockley, Read, Hall (SRH) recombination statistics, recombination current at the interface within the emitter-base space charge region can be expressed as [1]:

$$
I_{B s . c .} \cong \frac{1}{2} q n_{i} P_{E} x_{d B} v_{\text {surf }} \exp \left(\frac{V_{E B}}{2 V_{T}}\right)
$$

where $q$ is electronic charge, $P_{E}$ is the emitter perimeter, $n_{i}$ is the intrinsic carrier concentration, $V_{T}$ is the thermal voltage, $v_{\text {surf }}$ is the surface recombination velocity (and a function of interface trap density), and $x_{d B}$ is the approximate length over which the recombination rate is maximized. In most bipolar devices, the effect of space-charge recombination is only apparent at very low levels of emitter-base voltage. However, space-charge 
recombination becomes one of the primary base current mechanisms as $v_{\text {surf }}$ increases with total dose [1-4]. A second mechanism for radiation induced excess base current is recombination current at the interface within the neutral base. Using SRH statistics, neutral base recombination current can be approximated as [11]:

$$
I_{B n . b .} \cong q P_{E} v_{\text {surf }} \int_{0}^{x_{B}} p(x) d x
$$

where $x_{B}$ is the neutral base width and $p(x)$ is the excess minority carrier concentration across the neutral base.

The emitter-tied field plate suppresses gain degradation by reducing recombination current in the space charge and neutral base regions. Fig. 6 demonstrates the effect of varying field plate lengths on excess base current. In this figure, the increase in base current due to the added interface parameters ( $S R V=10^{4} \mathrm{~cm} / \mathrm{s}$ and $\mathrm{N}_{\mathrm{OX}}=10^{11} \mathrm{~cm}^{-2}$ ) for three field plate lengths is plotted versus emitter-base voltage. For voltages levels below $0.6 \mathrm{~V}$ where space-charge recombination current tends to dominate base current response, excess current is reduced as much as 50 percent when field plates extend over the active base. This is because a field plate over the space charge region will significantly reduce $x_{d B}$ in equation (1). The curves in Fig. 6 also show that there is no apparent difference between the effects of the $1.0 \mu \mathrm{m}$ and $2.0 \mu \mathrm{m}$ plates.

For emitter-base voltage levels above $0.6 \mathrm{~V}$, where neutral base recombination is dominant, the $2.0 \mu \mathrm{m}$ plate reduces excess base current as much as 65 percent over the device without a field plate. The $1.0 \mu \mathrm{m}$ plate has less effect, reducing current by 50 percent. The field plate over the active base suppresses neutral base recombination by decreasing the minority carrier concentration $p(x)$ in equation (2). The $2.0 \mu \mathrm{m}$ field plate has the greatest effect because it extends almost entirely across the neutral base. The field plate suppresses recombination current by reducing the number of carriers recombining with radiation-induced interface traps in both the emitter-base space charge and neutral base regions. Based on the results presented in this paper, future screening programs for hardness-assurance may find it beneficial to include the relatively inexpensive analysis of device or circuit metallization layouts. Moreover, future transistor designs for the radiation environment may benefit from simply extending the emitter contact across the active base region to form an emitter-tied field plate, thereby enhancing the LPNP device's resistance to radiation-induced gain degradation.

\section{REFERENCES}

[1] D.M. Schmidt, A. Wu, R.D. Schrimpf, D.M. Fleetwood, and R.L. Pease,"Modeling Ionizing Radiation Induced Gain Degradation on the Lateral PNP Bipolar Junction Transistor," IEEE Trans. Nucl. Sci., vol. 43, pp. 3032-3039, 1996.

[2] A. Wu, R.D. Schrimpf, H.J. Barnaby, D.M. Fleetwood, R.L. Pease, and S.L. Kosier,"Radiation-Induced Gain Degradation in Lateral PNP BJTs with Lightly and Heavily Doped Emitters," IEEE Trans. Nucl. Sci., vol. 44, pp. 1914-1921, 1997.

[3] S.L. Kosier, R.D.Schrimpf, D.M. Fleetwood, M. DeLaus, R.L. Pease, W.E. Combs, A Wei, and F.Chai, "Charge Separation for Bipolar Transistors," IEEE Trans. Nucl. Sci., vol. 40, pp. 1276-1285, 1993.

[4] S.L. Kosier, A. Wei, R.D.Schrimpf, D.M. Fleetwood, and M. DeLaus,"Physically Based Comparison of Hot-Carrier Induced and Ionizing Radiation Degradation in BJTs," IEEE Trans. Electron Dev., vol. 42, pp. 436-444, 1995.

[5] D.M. Fleetwood, L.C. Riewe, J.R. Schwank, S.C. Witczak, and R.D. Schrimpf, "Radiation Effects at Low Electric Fields in Thermal, SIMOX, and Bipolar-Base Oxides," IEEE Trans. Nucl. Sci., vol. 43, pp. 2537-2546, 1996.

[6] A.R. Hart, J.B. Smyth Jr., V.A.J. van Lint, D.P. Snowden, and R.E. Leadon, "Hardness Assurance Considerations for Long Term Ionizing Radiation Effects on Bipolar Transistors," IEEE Trans. Nucl. Sci., vol. 25, pp. 1502-1507, 1978

[7] V.G.K. Reddi, "Influence of Surface Conditions on Silicon Planar Transistor Gain," Solid-State Electron., vol. 10, pp. 305-334, 1967.

[8] H.E. Boesch Jr. and T.L. Taylor, "Charge and Interface State Generation in Field Oxides," IEEE Trans. Nucl. Sci., vol. 31, pp. 1273-1279, 1984.

[9] A.H. Johnston and R.E. Plaag, "Models for Total Dose Degradation of Linear Integrated Circuits," IEEE Trans. Nucl. Sci., vol. 34, pp. 1474-1480, 1987.

[10]E.W. Enlow, R.L. Pease, W.E. Combs, R.D. Schrimpf, and R.N. Nowlin, " Response of Advanced Bipolar Processes to Ionizing Radiation," IEEE Trans. Nucl. Sci., vol. 38, pp. 1342-1351, 1991.

[11]D. A. Neamen, Semiconductor Physics and Devices. Boston: Irwin, 1992.

[12] A.S. Grove and D.J. Fitzgerald, "Surface effects on p-n junctions: Characteristics of surface space-charge regions under non-equilibrium conditions," Solid-State Electron., vol. 9, pp. 783-806, 1966. 


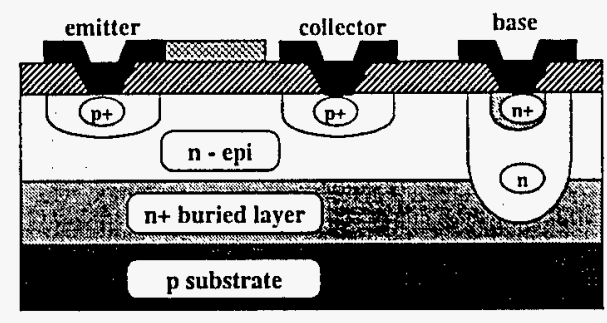

WIA. oxide metal contact field plate

Fig. 1 Representational cross-section of LPNP device with emitter-tied field plate.

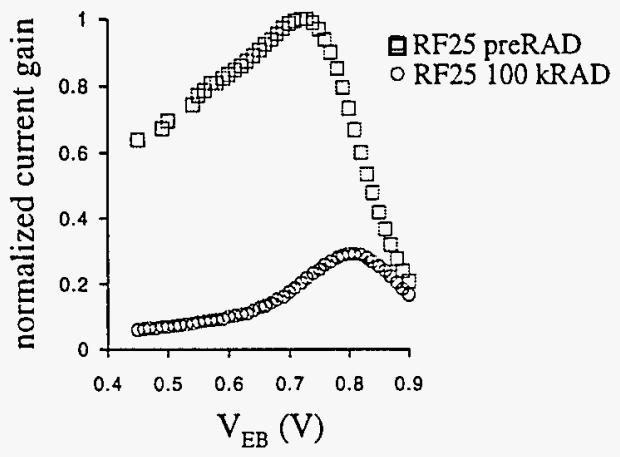

(a)

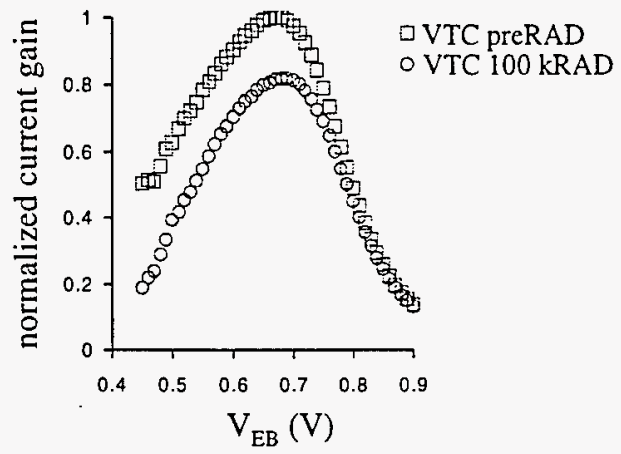

(b)

Fig. 2 Normalized current gain prior to radiation exposure and after $100 \mathrm{krad}\left(\mathrm{SiO}_{2}\right)$ total dose for: a) RF25 devices irradiated at $167 \mathrm{rad}\left(\mathrm{SiO}_{2}\right) / \mathrm{s}$ and b) VTC devices irradiated at $83 \mathrm{rad}\left(\mathrm{SiO}_{2}\right) / \mathrm{s}$.

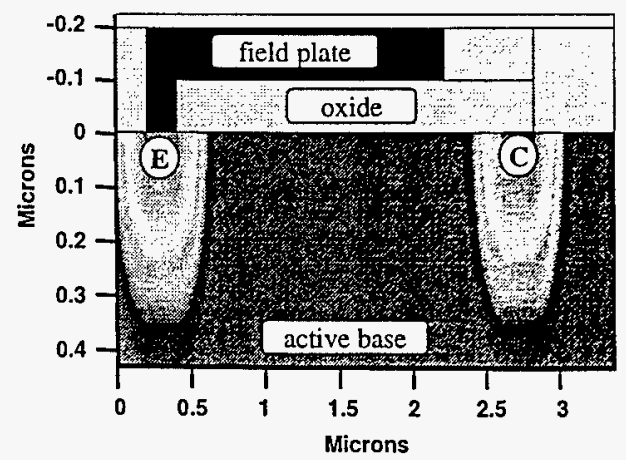

Fig. 3 Cross-section of LPNP simulation test structure with emitter-tied field plate.

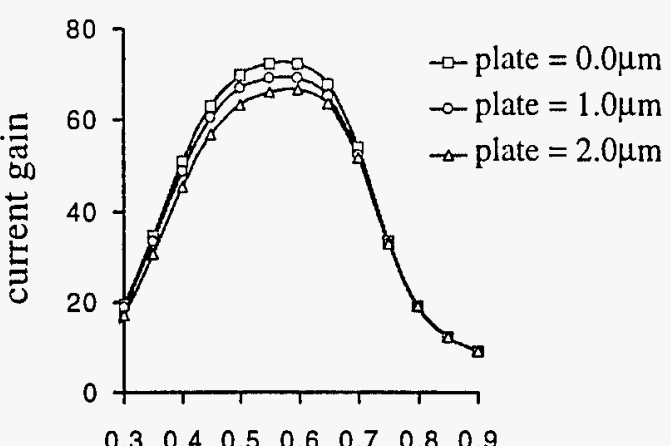

$$
\mathrm{V}_{\mathrm{EB}}(\mathrm{V})
$$

Fig. 4 Simulated current gain for pre-irradiation LPNP model with varying field plate lengths.

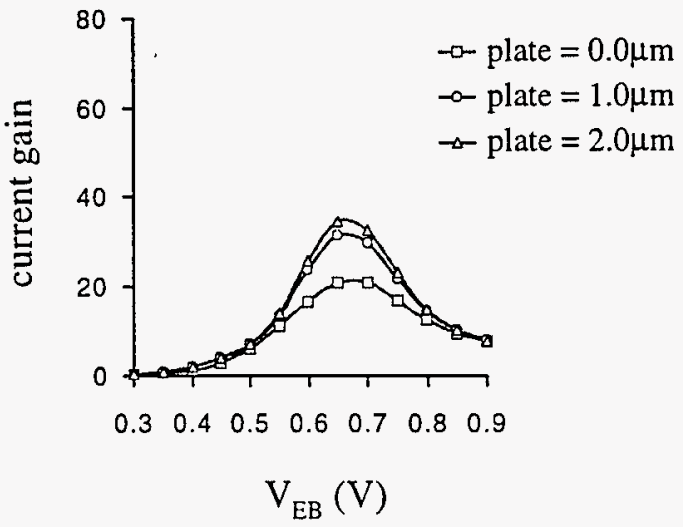

Fig. 5 Simulated current gain for post-irradiation LPNP model with varying field plate lengths.

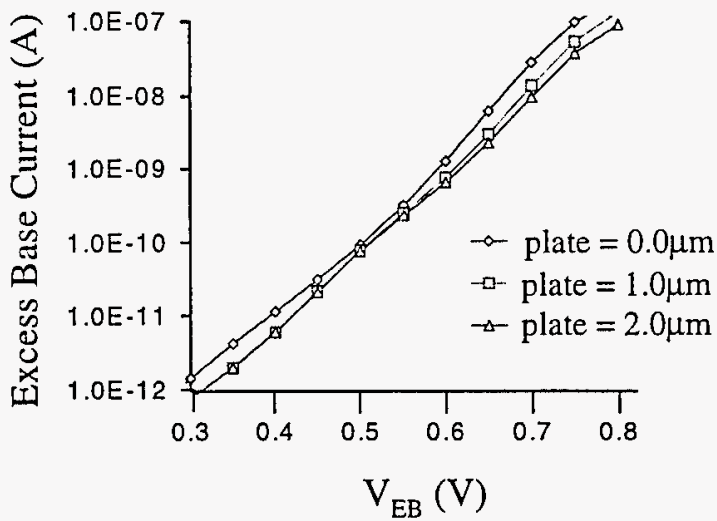

Fig. 6 Simulated excess base current for varying field plate lengths. 


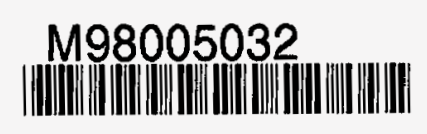

Report Number (14) $5 A N D--98-0625 C$
CONF- $980705-$

Publ. Date (11) 199803
Sponsor Code (18) $10 E / M A$,
UC Category (19) $4 \mathrm{C}-900$,

\section{0}

